\title{
Response to proposal for a novel cancer drug pricing model
}

\section{Toon van der Gronde (iD, Hubertus G. Leufkens and Toine Pieters}

In their recent News \& Views article (Sustainability and affordability of cancer drugs: a novel pricing model. Nat. Rev. Clin. Oncol. 15, 405-406 (2018)) $)^{1}$, Uyl-De Groot and Löwenberg outline a new universal algorithm for setting the price of new drugs in oncology. Their ambitious proposal is intended to standardize a complicated and fragmented pricing process. The international drug market is dynamic and diverse ${ }^{2}$. Having a universal pricing mechanism could indeed be helpful in addressing the imbalances in drug pricing and improve access to medicines for many patients, including those with nononcological diseases. However, we would like to point out some thoughts on the proposed algorithm.

First, the scope of the algorithm seems to be limited to the USA and European Union (EU). Outrage about high drug prices and the associated limitations in access to potentially life-saving drugs are certainly not limited to these regions. Importantly, most of the economic and population growth in the next few decades, and subsequently the burden of disease, will be in developing areas of Asia and Africa. Therefore, to have a truly universal framework, these regions would have to be included. Furthermore, the correction factors for countries with varying levels of economic development should be based on a more sophisticated tool than the one proposed, based on gross domestic product per capita equivalent costs per disability-adjusted life year averted ${ }^{3}$.

Second, all parameters of the algorithm carry inherent risks of unwanted stakeholder behaviour, by both industry and payers. Acknowledging research and development (R\&D) costs seems reasonable, but this does not necessarily encourage improvements in clinical benefit. Even if the price of R\&D could be calculated objectively (the analysis cited by the authors ${ }^{4}$ is already highly debated ${ }^{5-7}$ ) and if governments succeed in transnationally coordinating the reimbursement of $\mathrm{R} \& \mathrm{D}$ costs, this could stimulate spending on $\mathrm{R} \& \mathrm{D}$ with very little incentive to bring meaningful improvements in the quality of life of patients.

Third, years left on patent is a parameter that provides ample opportunity for interestbased manoeuvring. Patents are frequently extended or renewed based on additional research efforts (such as extension of a patent after the completion of research in paediatric populations ${ }^{8,9}$ ) or changes in formulation, thus making estimates of remaining patent life unreliable. Furthermore, the 3-year evaluation period would incentivize producers to delay the launch of any new drug indications until after the recalculation, so that the target population is smaller and, therefore, the accepted drug price will be higher. Similarly, such measures would encourage companies to introduce new indications as quickly as possible following recalculation, in order to maximize financial benefit at the expense of both patients and health-care providers.

In conclusion, the development of a universal framework to guide something as complex as the drug pricing process is an admirable undertaking that merits ample debate. Any model that is adopted will drive stakeholder behaviour towards optimally satisfying their own interests. The key challenge is, therefore, to align the interests of stakeholders with those of the general public. Even the best available model, value-based pricing, can lead to unacceptable outcomes and limitations in access. This scenario is seen in the recent debate around hepatitis $C$ products, which have superb clinical benefits but are unaffordable for the population ${ }^{2}$. The proposed algorithm is a laudable initiative, but requires careful assessment to ensure the provision of a framework that helps streamline the pricing process and also ensures equal access to new drugs for patients around the world.
There is a reply to this letter by Uyl-de Groot, C. A. \& Löwenberg, B. Nat. Rev. Clin. Oncol. https://doi.org/10.1038/s41571-0180063-6 (2018).

Toon van der Gronde (D) ${ }^{1,2}$, Hubertus G. Leufkens ${ }^{2}$ and Toine Pieters ${ }^{2 *}$ ${ }^{\prime}$ AstraZeneca, Cambridge, United Kingdom. ${ }^{2}$ Utrecht Institute for Pharmaceutical Sciences (UIPS) Division of Pharmacoepidemiology and Clinical Pharmacology, Utrecht University, Utrecht, Netherlands.

*e-mail: t.pieters@uu.nl https:/doi.org/10.1038/s41571-018-0062-7

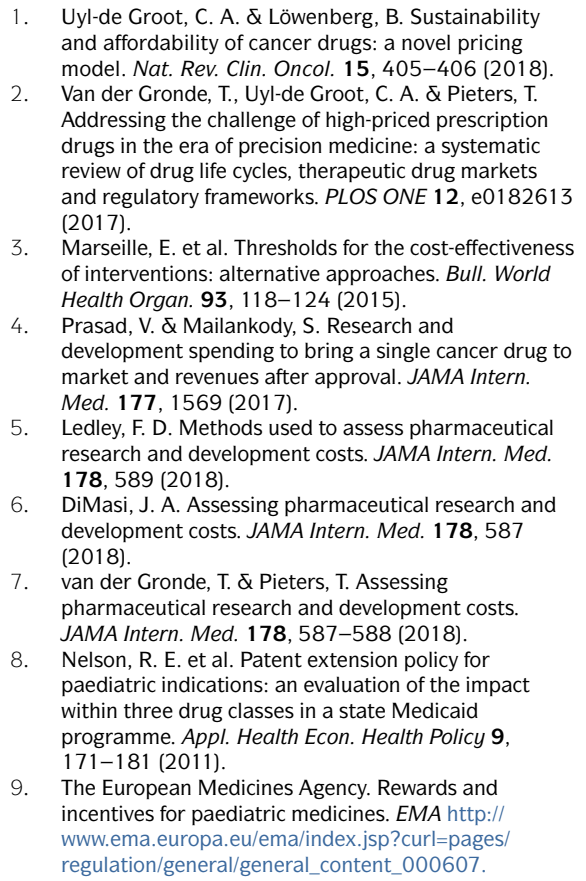
and affordability of cancer drugs: a novel pricing model. Nat. Rev. Clin. Oncol. 15, 405-406 (2018). 2. Van der Gronde, T., Uyl-de Groot, C. A. \& Pieters, T. Addressing the challenge of high-priced prescription drugs in the era of precision medicine: a systematic review of drug life cycles, therapeutic drug markets and regulatory frameworks. PLOS ONE 12, e0182613 (2017).

3. Marseille, E. et al. Thresholds for the cost-effectiveness of interventions: alternative approaches. Bull. World Health Organ. 93, 118-124 (2015).

4. Prasad, V. \& Mailankody, S. Research and development spending to bring a single cancer drug to market and revenues after approval. JAMA Intern. Med. 177, 1569 (2017).

5. Ledley, F. D. Methods used to assess pharmaceutical research and development costs. JAMA Intern. Med. 178, 589 (2018).

6. DiMasi, J. A. Assessing pharmaceutical research and development costs. JAMA Intern. Med. 178, 587 (2018).

7. van der Gronde, T. $\&$ Pieters, T. Assessing pharmaceutical research and development costs. JAMA Intern. Med. 178, 587-588 (2018).

8. Nelson, R. E. et al. Patent extension policy for paediatric indications: an evaluation of the impact within three drug classes in a state Medicaid programme. Appl. Health Econ. Health Policy 9 , 171-181 (2011)

9. The European Medicines Agency. Rewards and incentives for paediatric medicines. EMA http:// www.ema.europa.eu/ema/index.jsp?curl=pages/ regulation/general/general_content_000607. jsp\&mid=WCOb01 ac0580925b 1 c (2018) .

\section{Competing interests}

T.v.d.G. works in a full-time position for AstraZeneca. AstraZeneca had no influence on any aspect of this manuscript. H.G.L. reports that he is the past chairman of Dutch Medicines Evaluation Board and past member of the European Medicines Agency (EMA) Committee for Medicinal Products for Human Use (CHMP), and scientific director of the Utrecht WHO Collaborating Centre for Pharmaceutical Policy and Regulation. This centre accepts no direct funding or donations from the pharmaceutical industry or other private parties. T.P. declares no competing interests.

\section{Reply to 'Response to proposal for a novel cancer drug pricing model'}

\section{Carin A. Uyl-de Groot and Bob Löwenberg}

We agree with the statement (in response to our original publication ${ }^{1}$ ) that the development of an improved framework for drug pricing requires streamlining of the process to ensure equal access of patients with new drugs (Response to proposal for a novel cancer drug pricing model. Nat. Rev. Clin. Oncol. https://doi.org/10.1038/s41571-018$0062-7)^{2}$. This requirement has been precisely the motivation for our entirely different approach in proposing a novel pricing system. Some potential practical impediments are also 